\title{
Mitochondriale Medizin
}

\section{Liebe Leserinnen, liebe Leser,}

Mitochondrien sind sehr alte lebende Strukturen, die aus der Verbindung von Urbakterien und Zellen hervorgingen. Der Begriff Mitochondrium für die 1856 erstmals konkret beschriebenen Zellorganellen in quergestreifter Muskulatur wird 1898 eingeführt. Mitochondrien finden sich in jeder Körperzelle (außer reifen Erythrozyten), sie sind die zellulären Kraftwerke, die das lebensnotwendige ATP generieren. Pro Zelle finden sich einige hundert bis zu mehreren tausend dieser Organellen. Zellen mit hohem Energiebedarf wie Herz- und Skelettmuskelzellen oder Hirnzellen besitzen mehr als 10000 Mitochondrien.

Die erste Erkrankung durch mitochondriale Dysfunktion wurde 1962 von Luft und Kollegen im Journal of Clinical Investigation beschrieben. Seit dieser Zeit hat das Interesse und auch die Forschung im Bereich Struktur und Funktion der Mitochondrien sowie das Wissen über die Bedeutung im Stoffwechsel und die ursächlichen Zusammenhänge mit vielen Gesundheitsstörungen dramatisch zugenommen. Organe sind in Abhängigkeit von ihrem Energiebedarf unterschiedlich stark von einer mitochondrialen Funktionsstörung betroffen. Herz- und Skelettmuskelzellen, Gehirn oder auch die Netzhaut reagieren daher viel ausgeprägter auf eine mitochondriale Insuffizienz.

Eine mitochondriale Dysfunktion ist gekennzeichnet durch verminderte ATP-
Syntheseleistung und damit verbundenem Energiedefizit einerseits und erhöhtem oxidativen Stress sowie vermehrter Mutation der mitochondrialen DNA (mtDNA) andererseits. Dies führt zu funktioneller Insuffizienz der betroffenen Zellen bzw. Gewebe und zu frühzeitigem Untergang und mutagener Veränderung. Muskelschwäche, rasche muskuläre Ermüdbarkeit, schlechte Trainierbarkeit oder Muskelschmerzen bei Belastung oder sogar in Ruhe können bei jungen Patienten u.U. erste Hinweise auf eine mitochondriale Dysfunktion sein.

Mitochondriale Erkrankungen auf dem Boden von mtDNA-Mutationen haben eine Prävalenz von ca. 10-20 pro 100000 . Dabei wird unterschieden zwischen ererbter Mutation (Beschwerden zeigen sich bereits im Kindesalter) und erworbenen Mutationen (Beschwerden entwickeln sich im Laufe des Lebens). Heute sind mehr als 200 Mutationen der mtDNA bekannt. Neben den durch einen ererbten Gendefekt in der mtDNA ausgelösten Erkrankungen wie Epilepsie, Optikusatrophie und Augenmuskellähmung mit Sehstörungen werden aber immer mehr im Laufe des Lebens erworbene Erkrankungen mit einer mitochondrialen Dysfunktion in ursächliche Verbindung gebracht. Dazu zählen u.a. neurodegenerative Erkrankungen wie M. Alzheimer oder M. Parkinson, kardiovaskuläre Erkrankungen, Diabetes mellitus oder auch Tumorerkrankungen.
Sowohl für die Diagnostik als auch für die Therapie stehen uns z.Z. noch nicht viele Möglichkeiten zur Verfügung und nicht alles, was in den Forschungsinstituten bereits üblich ist, lässt sich im klinischen Alltag nutzen. Ein ganz wesentlicher Schritt ist es aber, an diese Zusammenhänge bereits zu denken.

In den Titelbeiträgen dieser Ausgabe stellen wir Ihnen grundlegende Mechanismen und Zusammenhänge sowie das toxische Potenzial einiger Arzneimittel im Hinblick auf die Mitochondrienfunktion dar. Darauf aufbauend zeigen wir in der Rubrik Praxistipps einige diagnostische und therapeutische Ansätze auf.

Die mitochondriale Medizin ist ein „aufregender“ Bereich und er wird sich zweifelsohne mit großer Dynamik entwickeln und uns neue Einsichten in die Entstehung, Diagnostik und Therapie chronischer Gesundheitsstörungen vermitteln.

\section{Ihre Herausgeber}
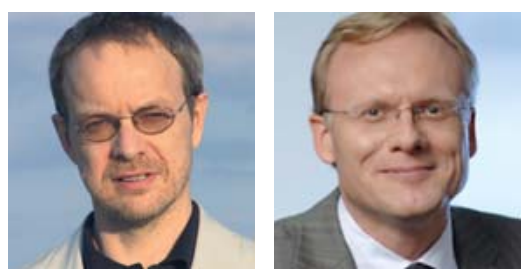

Dr. med. Hans-Peter Friedrichsen
Apotheker Uwe Gröber 